\title{
Secondary Sarcomatous Transformation in Mature Cystic Teratoma: An Uncommon Cause of Acute Abdomen
}

\author{
Nadia Shirazi ${ }^{1^{*}}$, Meena Harsh ${ }^{1}$, Dev Nanda Chaudhary ${ }^{2}$ and Divya Mahajan ${ }^{1}$ \\ ${ }^{1}$ Department of Pathology, Himalayan Institute of Medical Sciences, Swami Rama Himalayan University, Dehradun, India \\ ${ }^{2}$ Department of Gynecology, Himalayan Institute of Medical Sciences, Swami Rama Himalayan University, Dehradun, India
}

"Corresponding author: Dr. Nadia Shirazi, Department of Pathology, B-IX-6, HIHT Campus, Jolly Grant, Dehradun-248140, Uttarakhand, India, Tel:91-9758376477; Email: shirazinadia@gmail.com

Received date: October 21, 2014; Accepted date: November 14, 2014; Published date: November 21, 2014

Copyright: ( $) 2014$ Nadia Shirazi, et al. This is an open-access article distributed under the terms of the Creative Commons Attribution License, which permits unrestricted use, distribution, and reproduction in any medium, provided the original author and source are credited.

\begin{abstract}
Malignant transformation in a mature cystic teratoma is rare and sarcomatous change is even rarer. We report a case of 57 year old post-menopausal woman who presented with tender abdominal mass and fever. She underwent Total Abdominal Hysterectomy with Bilateral Salpingo-oophorectomy. Left ovary showed a mature cystic teratoma with solid areas. Histopathology of these solid areas showed a high grade spindle cell sarcoma which exhibited Vimentin and SMA positivity on Immunohistochemistry and was thus diagnosed as leiomyosarcoma.
\end{abstract}

In most instances malignant transformation cannot be diagnosed pre-operatively. Most of these tumors are considered resistant to radiation and systemic chemotherapy and carry a poor prognosis.

Keywords: Mature cystic teratoma; Malignant transformation; Immunohistochemistry; High grade sarcoma; Leiomyosarcoma

\section{Abbreviations:}

CEA: Carcinoembryonic Antigen; AFP: Alpha Fetoprotein; CA Carbohydrate Antigen, HCG: Human Chorionic Gonadotropin; LDH: Lactate Dehydrogenase; MRI: Magnetic Resonance Imaging

\section{Introduction}

Malignant transformation in a mature cystic teratoma (MCT) is extremely rare with an incidence of 0.17 to $2 \%$ of all cases [1]. Even if such a transformation occurs it is usually a squamous cell carcinoma. Sarcomatous transformation is very unusual and carries a particularly grim prognosis. There are very few published studies on sarcomatous transformation in ovarian teratomas [2] therefore this case will add to the knowledge of both pathologists and gynaecologists and thereby helping in an early diagnosis.

\section{Case Report}

A 57 year old obese post-menopausal woman came to the gynaecology OPD with chief complains of pain in left hypochondrium since 1 day. The pain was deep, aching and non-radiating. It was not associated with any genito-urinary complains or bleeding per vaginum $(\mathrm{P} / \mathrm{V})$. Patient had high grade fever associated with chills since 30 days for which she had consulted a local practitioner who gave symptomatic treatment. Patient was a hypertensive and was on irregular medications since 4-5 years. Per Abdomen (P/A) findings revealed a large mass palpable of 22 weeks gravid uterus size, firm in consistency with well defined margins. It was highly tender on palpation. On palpating right side of abdomen nothing was felt . P/V and Per Speculum (P/S) findings showed normal looking cervix.
Investigations: Haemogram was within normal limits with $76 \%$ neutrophilia. ESR was elevated ( $48 \mathrm{~mm} / 1$ st hour). Widal and Malarial Parasite (MP) tests were negative. Her CA-125 was within normal limits (14.6). AFP, CEA, HCG and LDH were normal. Ultrasound abdomen showed a massive solid and cystic mass arising from left adnexa. MRI pelvis showed a large teratodermoid cyst. A Total Abdominal Hysterectomy with Bilateral Salpingo-Oophorectomy was planned. Per-operatively a huge left ovarian cyst was seen measuring $20 \times 15 \mathrm{~cm}$. The mass had undergone torsion and was twisted on its pedicle twice. Uterus was visualised after removing the cyst. It was soft and atrophic. Right side tube and ovary were normal looking and were removed. The specimen was sent for histopathological examination.

Grossly, uterus with cervix with right tube and ovary showed no significant pathology. Left ovary showed a partly solid and partly cystic mass measuring $16 \times 15 \times 10 \mathrm{~cm}$. Capsule was ruptured and the outer serosal aspect was covered with fibrinous exudates and cheesy material. The contents of the cyst were cheesy material admixed with hair (Figure 1).

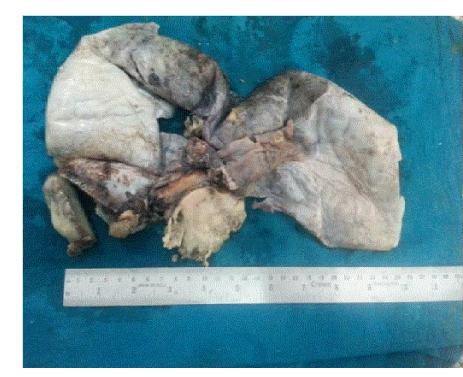

Figure 1: Opened up specimen of left ovarian cyst.

On microscopic examination left ovary showed a partly cystic mature teratoma (Photomicrograph 1). 
Citation: Shirazi N, Harsh M, Chaudhary DN, Mahajan D (2014) Secondary Sarcomatous Transformation in Mature Cystic Teratoma: An Uncommon Cause of Acute Abdomen. J Integr Oncol 3: 122. doi:10.4172/2329-6771.1000122

Page 2 of 3

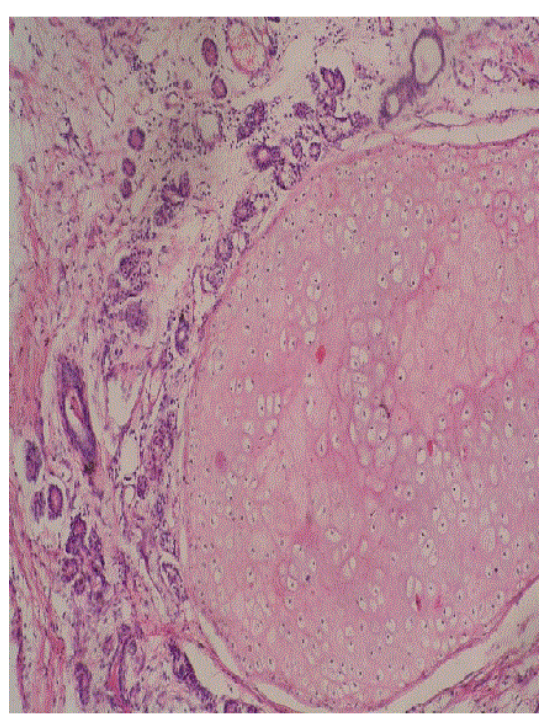

Photomicrograph 1: 10x10X: H\&E: Mature Cystic Teratoma with cartilage.

Sections from solid area showed a cellular high grade spindle cell sarcoma. Tumor was arranged in fascicles, cells had spindle shaped nuclei with indistinct cell borders. Mitotic figures were 8-10/10 high power field (Photomicrograph 2).

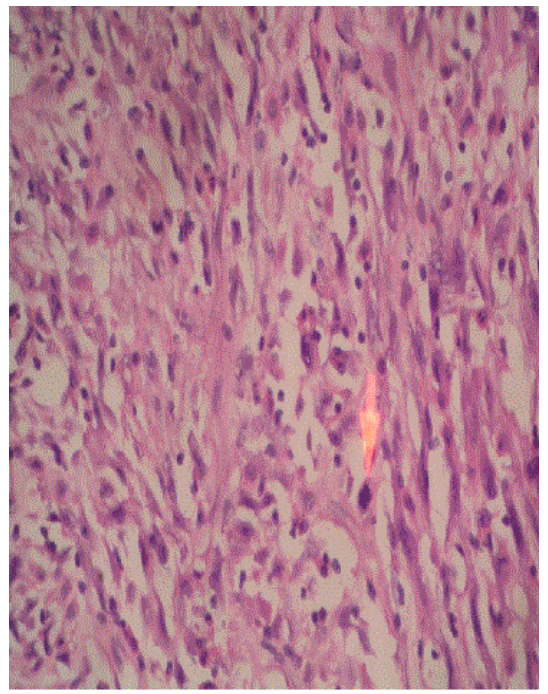

Photomicrograph 2: 10x10X: H\&E: Spindle cell sarcoma with brisk mitotic activity.

Large areas of necrosis were seen. Tumor was limited to the left ovary and capsular infiltration was not seen. A diagnosis of mature cystic teratoma with secondary malignant transformation into high grade sarcoma was rendered (FIGO Stage I). On Immunohistochemistry the tumor was positive for Vimentin (Photomicrograph 3) and Smooth Muscle Actin (Photomicrograph 4) however negatuve for Desmin and Cytokeratin. A diagnosis of leiomyosarcoma was confirmed on the basis of these findings.

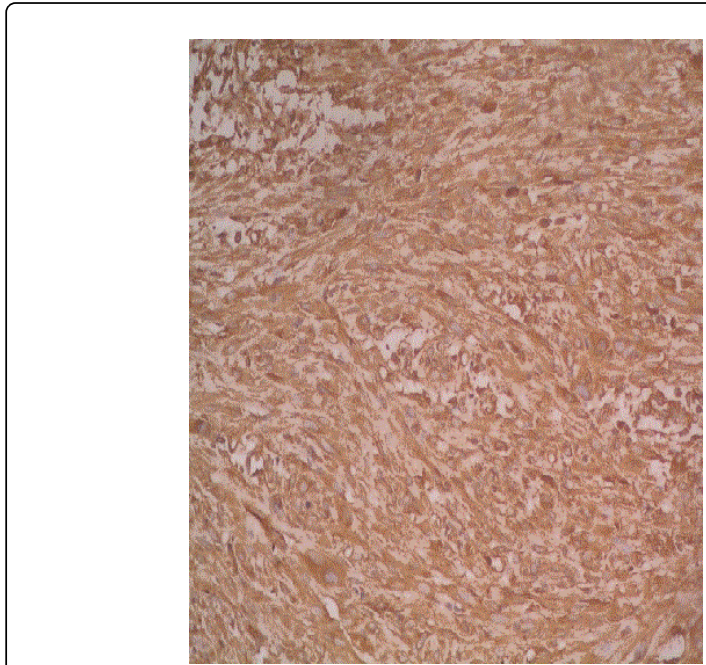

Photomicrograph 3: 10x10X: Immunohistochemistry -Vimentin: Strong cytoplasmic positivity

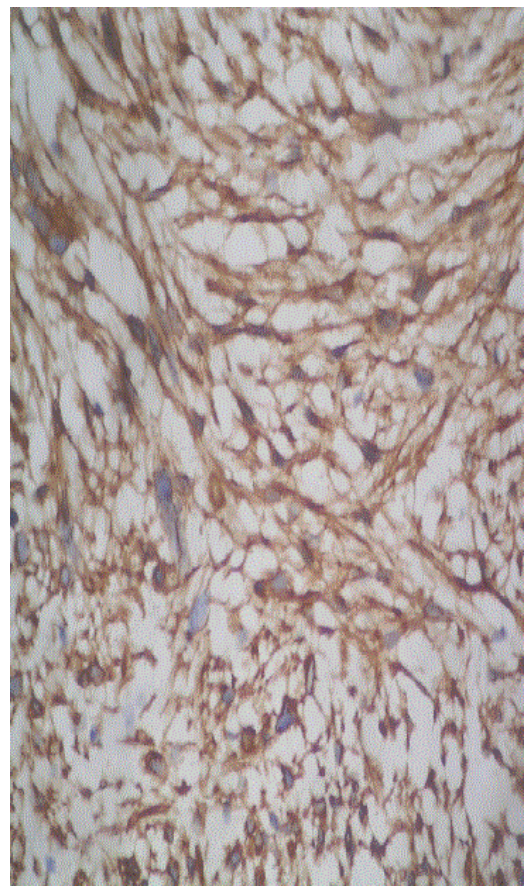

Photomicrograph 4: 40x10X Strong SMA positivity

Patient was given 2 cycles of chemotherapy (CAP regimeCyclophosphamide $500 \mathrm{mg} / \mathrm{m}^{2}$, Adriamycin $50 \mathrm{mg} / \mathrm{m}^{2}$, Cis-platinum $50 \mathrm{mg} / \mathrm{m}^{2}$ ). Patient tolerated chemotherapy well and was discharged to come again for 5-6 courses of Chemotherapy at monthly interval. 
Citation: Shirazi N, Harsh M, Chaudhary DN, Mahajan D (2014) Secondary Sarcomatous Transformation in Mature Cystic Teratoma: An Uncommon Cause of Acute Abdomen. J Integr Oncol 3: 122. doi:10.4172/2329-6771.1000122

Page 3 of 3

\section{Discussion}

Germ cell tumors account for approximately $30 \%$ of all ovarian tumors. 95\% of all Germ Cell tumors are dermoid cysts. According to WHO, malignancy arising within a mature cystic teratoma is a rare complication seen in $<1-2 \%$ cases, mostly reported in postmenopausal women (mean age 51-62 years). The most common malignant change in cystic teratoma is Squamous cell carcinoma (75\%), followed by carcinoid tumor, adenocarcinoma and melanomas. Sarcomas account for $8 \%$ malignancies in dermoid cysts [2]. The frequency of malignant change increases with increasing age, rising to $19 \%$ in women after menopause [3]. Risk factors for malignancy include age $>45$ years, tumor diameter $>10 \mathrm{~cm}$ and rapid growth [4] The prognosis of patients with malignant transformation in teratoma is very poor, with a 5-year survival rate of only $15-30 \%$ [4]. However, the prognosis is considered to be better if the tumor is limited to one ovary with an intact capsule and not adhered to surrounding structures [4].

According to a retrospective series by Park et al., squamous cell carcinoma was the most common histologic type of malignant transformation in MCT comprising of $75 \%$ of cases [5]. Whereas in a study by Malagon et al., the most common sarcomatous component to arise in germ cell tumors was embryonal rhabdomyosarcoma (29/46) followed by angiosarcoma (6/46), leiomyosarcoma (4/46) and undifferentiated sarcoma (3/46) [6]. Savitchi et al. reported co-existent squamous cell carcinoma and pleomorphic sarcoma arising in a mature cystic teratoma [7]. Tumor markers like CEA may not be elevated or else a false positive elevation can be seen in $14.3 \%$ cases which turn out to be normal in the repeated study [8]. Hence it can be stated that tumor markers do not play a significant role in giving a preoperative diagnosis in all ovarian tumors. These tumors respond poorly to radiotherapy and systemic chemotherapy and carry a very poor prognosis with most patients presenting as early recurrence or death within 1 year of initial diagnosis [9].

\section{Conclusion}

We report a very rare occurrence of sarcomatous malignant transformation in surgically resected ovarian mass suspected to be mature cystic teratoma and presenting as acute abdomen. Neither imaging studies nor tumor marker levels can accurately diagnose such a malignant transformation pre-operatively. Hence all the dermoid cysts should be examined histologically by giving multiple sections with particular focus on solid areas along with the aid of immunohistochemistry in case of poorly differentiated tumors. This will result in more such cases being identified and thus treated at an early stage.

\section{References}

1. Young RH, Cement PB, Scully RE (2004) Sex cord stromal, steroid cell and germ cell tumors of the ovary. Sternberg's Diagnostic Surgical Pathology (4th edtn). Lippincott Williams \& Wilkins, London, UK.

2. Nogales F, Talerman A, Kubik-Huch RA, Tavassoli FA, DevonassovxShisheboran M, et al. (2003) Germ cell tumors In World Health Organization Classification of tumors. Pathology and Genetics of Tumors of the Breast and Female Genital Organs 2: 163-175.

3. Anderson MC. Tumors of the ovary: Germ cell tumors. Chapter 20 In Symmers St.W.C. Systemic Pathology by 38 authors. Gynaecological Pathology (2nd edtn).

4. Al-Rayyan ES, Duqoum WJ, Sawalha MS, Nascimento MC, Pather S, et al. (2009) Secondary malignancies in ovarian dermoid cyst. Saudi Med J 30: 524-528.

5. Park JY, Kim DY, Kim JH, Kim YM, Kim YT, et al. (2008) Malignant transformation of mature cystic teratoma of the ovary: experience at a single institution. Eur J Obstet Gynecol Reprod Biol 141: 173-178.

6. Malagon HD, Valdez AM, Moran CA, Suster S (2007) Germ cell tumors with sarcomatous components: a clinicopathologic and immunohistochemical study of 46 cases. Am J Surg Pathol 31: 1356-1362.

7. Savitchi E, Rao S (2012) Squamous cell carcinoma and pleomorphic sarcoma (MFH) arising in a mature cystic teratoma of the ovary. Int J Gynecol Pathol 31: 443-446.

8. Yamaguchi K, Mandai M, Fukuhara K, Higuchi T, Hamanishi J, et al. (2008) Malignant transformation of mature cystic teratoma of the ovary including three cases occurring during follow-up period. Oncol Rep 19: 705-711.

9. Donadio AC, Motzer RJ, Bajorin DF, Kantoff PW, Sheinfeld J, et al. (2003) Chemotherapy for teratoma with malignant transformation. J Clin Oncol 21: 4285-4291. 